\title{
A virtual-clinic pathway for patients referred from a national diabetes eye screening programme reduces service demands whilst maintaining quality of care
}

\author{
Livia Faes ${ }^{1,2} \cdot$ Dun Jack Fu $\mathbb{D}^{1} \cdot$ Josef Huemer $\mathbb{D}^{1,3} \cdot$ Christoph Kern $\mathbb{D}^{1,4} \cdot$ Siegfried K. Wagner $^{1} \cdot$ Sandro Fasolo $^{1}$.

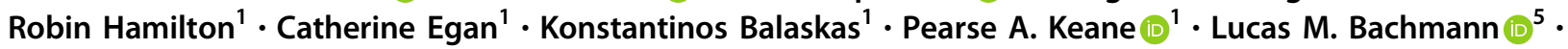 \\ Dawn A. Sim (1D)
}

Received: 25 February 2020 / Revised: 27 September 2020 / Accepted: 16 October 2020 / Published online: 30 October 2020

(c) The Author(s), under exclusive licence to The Royal College of Ophthalmologists 2020

\begin{abstract}
Aim To evaluate the potential of an integrated virtual medical retina clinic in secondary care for diabetic patients screened and referred by the UK National Diabetic Eye Screening Program (DESP).

Methods This retrospective cohort study included diabetic patients referred by the DESP to either a virtual or a traditional doctor's appointment (face-to-face, F2F) at the Moorfields Eye Hospital NHS Foundation Trust (London, UK) between January 2015 and December 2018. The primary outcome was the proportion of patients that qualified for a virtual-clinic appointment according to hospital guidance. Secondary outcomes included the rate of attendance, mean time from DESP referral to initial hospital appointment, mean time-to-discharge and -to-treatment of either panretinal photocoagulation or intravitreal injection of anti-vascular endothelial growth factor.

Results We included 12,563 patients in this study. While 8833 patients $(70.7 \%)$ would have qualified for a virtual appointment according to local triage guidance, only $2306(18.4 \%)$ were referred to a virtual consultation due to capacity constraints. For routine referrals, mean time to the first hospital appointment was 66.9 days with a standard deviation of \pm 35.9 and $80.9 \pm 44.4$ days for a virtual and a F2F consultation, respectively. The mean time from referral to discharge to community was $71.7 \pm 30.8$ and $86.3 \pm 37.0$ days for a virtual and a F2F consultation, respectively. We did not observe a statistically significant difference in the mean time-to-treatment in the sub-cohort that required intravitreal therapy for maculopathy (virtual clinics: $220.7 \pm 84.8$; F2F: 178.0 days \pm 80.7 ; $p$ value $>0.05$ ). Moreover, we observed a non-inferior attendance rate in virtual as compared to F2F clinics.

Conclusion A significant proportion of diabetic patients referred to a F2F clinic could initially be managed in a virtual clinic. Increasing the adoption of virtual clinics in the management of diabetic patients that do not need long-term management or monitoring in secondary services may help alleviate service demands without diminishing quality of clinical care. Collectively, our analyses suggest that virtual consultations are a faster and clinically appropriate alternative for a substantial proportion of diabetic patients.
\end{abstract}

These authors contributed equally: Livia Faes, Dun Jack Fu

Supplementary information The online version of this article (https:// doi.org/10.1038/s41433-020-01240-z) contains supplementary material, which is available to authorized users.

Dawn A. Sim

dawnsim@nhs.net

1 Moorfields Eye Hospital NHS Foundation Trust and UCL Institute of Ophthalmology, London, UK

2 Department of Ophthalmology, Cantonal Hospital Lucerne, Lucerne, Switzerland

\section{Introduction}

Diabetes mellitus is estimated to affect more than 693 million people by 2045 worldwide [1]. More than a third of those patients will develop diabetic eye disease, of which

3 Vienna Institute for Research in Ocular Surgery, A Karl Landsteiner Institute, Hanusch Hospital, Vienna, Austria

4 Department of Ophthalmology, University Hospital of Munich (LMU), Munich, Germany

5 Medigntion, Zürich, Switzerland 
$10 \%$ will develop visual impairment. Early identification of diabetic eye disease is crucial in the prevention of sight deterioration [2, 3]. In 2003, the United Kingdom (UK) National Health Service (NHS) launched a nationwide diabetic eye screening program (DESP) aimed at reducing the risk of sight loss through early detection and treatment. The DESP offers an annual screening appointment to all people with diabetes aged 12 years or older. Here, colour fundus photography takes place in the primary care setting and identifies patients that may require secondary-carebased hospital eye services (HES) [4, 5]. In secondary care, patients undergo assessment for whether: (i) intervention (panretinal photocoagulation (PRP), intravitreal injections, surgery) or active monitoring (more frequent than annual) is indicated; or (ii) whether they can safely be discharged back to the DESP [4].

Following the implementation of the screening programme, 2009 marked the first year within five decades in which diabetic retinopathy was no longer the leading cause of blindness in England and Wales [2, 3]. Consequently, over two million diabetic patients were screened and over 60,000 (about 3\% were referred to HES in 2015 alone [6]. The introduction of the DESP has increased referrals to HES by $30 \%$ between 2010 and 2015. This has massively strained the capacity of HES to deliver high quality ophthalmic care [6, 7]-a problem not exclusive to the UK [8]. Hence, there is a worldwide need for efficient review of the relentlessly growing numbers of patients referred to secondary care. Ideally, such a review would allow rapid identification of patients that can be safely discharged to and monitored in primary care [9, 10].

The Royal College of Ophthalmologists defines virtual clinics as patient-clinician consultations in which the faceto-face $(\mathrm{F} 2 \mathrm{~F})$ interaction is removed. Broadly, these can be either synchronous (interaction between patient and clinician occurs in real time e.g., via teleconferencing) or asynchronous; wherein patient examination and clinician assessment are separated in both time and space. Both are key innovations that can enhance the efficiency of referral review. In particular time-independent review of patients, which allows additional flexibility for both physician and the patient by overcoming the need for patient-clinician schedules to overlap. Patients with diabetes are ideally suited for virtual consultations as retinal imaging including optical coherence tomography (OCT) and colour fundus photography are the foundation of modern retinal examination, and can be digitally stored and reviewed remotely [11, 12]. Virtual clinics in other ophthalmologic subspecialities have demonstrably reduced patient journey time, allowing for reduced waiting times and more patients to be monitored [13]. Accordingly, virtual medical retinal consultations were integrated into the medical retina service of Moorfields Eye Hospital (MEH) NHS Foundation Trust in 2015 [14, 15]. The MEH is a tertiary eye care hospital providing secondary care in this instance.

At present, DESP referrals to $\mathrm{MEH}$ are invited to an initial assessment at a traditional F2F or virtual clinic. A proportion of referrals is directly triaged to F2F, either those identified by the: (i) DESP to likely to require intervention i.e., proliferative retinopathy, best-corrected visual acuity (VA) below 6/18 Snellen (61 Early Treatment Diabetic Retinopathy [ETDRS] letters); or (ii) triaging MEH ophthalmologist as unsuitable for a virtual consultation (ungradable fundus image in secondary care, vulnerable adult, lens or media opacities, pregnancy, or requirement of interpreter services). Patients are otherwise indiscriminately assigned to either F2F or VC according to booking availability (Fig. 1).

In this study, we evaluated the potential of a combined F2F- and virtual-clinics system at the MEH by investigating the proportion of patients eligible for an initial assessment in the virtual setting. We also sought to collect preliminary evidence for the efficiency, safety, patient acceptance, and accessibility of the service through assessment of mean time from referral to first HES appointment, mean time-todischarge, mean time-to-treatment, the rate of attendance, and social and economic deprivation indices.

\section{Methods}

\section{Study setting and cohort selection}

All diabetic patients ( $n=16,224)$ referred by the DESP to secondary care at MEH NHS Foundation Trust (London, UK) between January 2015 and December 2018 were eligible. The cutoff date of January 2015 was chosen as it marks the period, in which the medical retina service at $\mathrm{MEH}$ integrated, a virtual-clinic option for the management of diabetic patients referred by the DESP. Patients that were re-referred after having been discharged from secondary care and patients without record of retinopathy, maculopathy, or best-corrected VA in both eyes at the secondary care appointment were excluded.

\section{Ethics}

We obtained approval by the Institutional Review Board of the hospital (ROAD17/031) for this study. An audit registration was completed (MEH-233). We comply with the Declaration of Helsinki and STROBE guidelines for the reporting of cohort studies [16].

\section{Study procedures}

The DESP invites all diabetics aged 12 years or over to an annual assessment detailed elsewhere [4] and summarised 


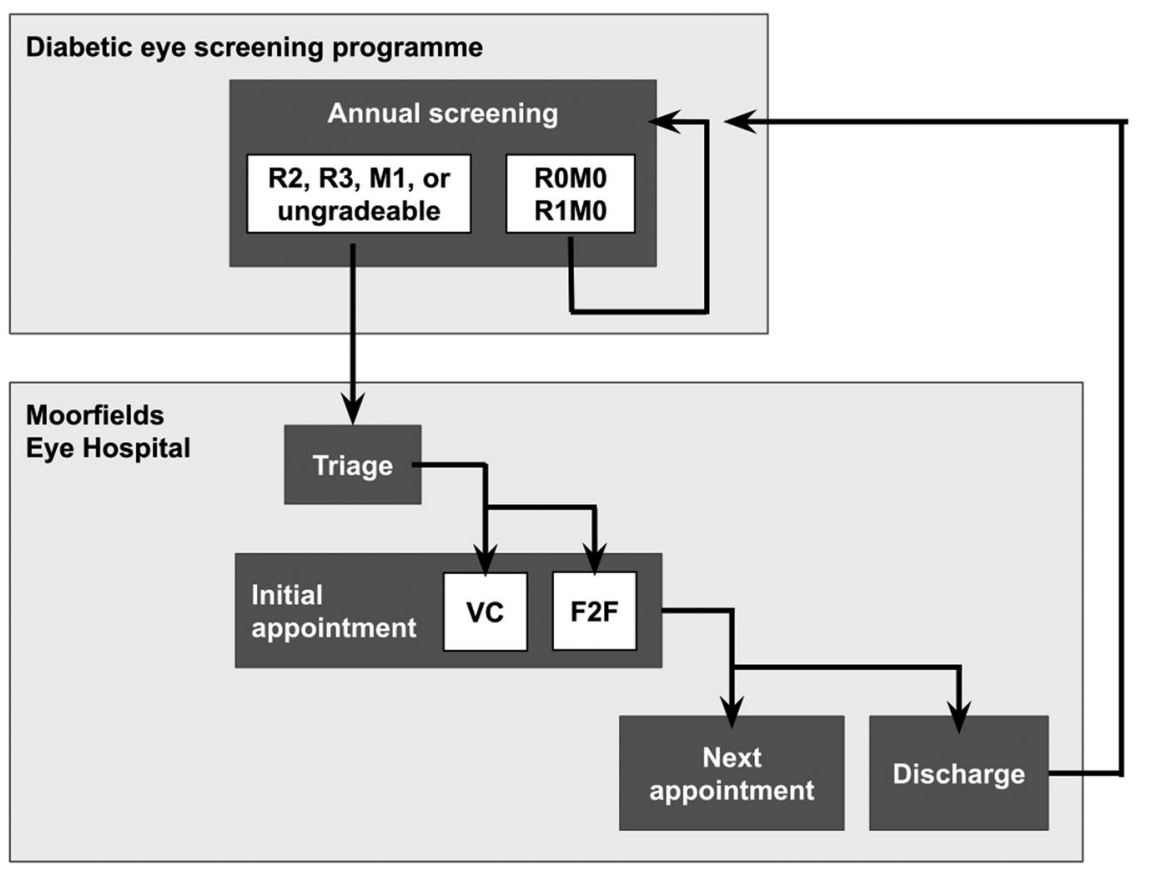

Fig. 1 Referral pathway from the NHS diabetic eye screening program (DESP) to Moorfields Eye Hospital. The DESP invites all diabetic patients aged 12 years or over to annual primary-care-based screening. Here, two-field fundus photography (one image centred on the macula and a second image centred on the optic disc) is acquired and graded according to the English Screening Programme for Diabetic Retinopathy standards (Supplementary Fig. 1). If criteria were met (R2, R3, R3, M1, or ungradable photo), patients are referred to hospital eye services and suspended from screening while under secondary care. Urgently referred patients (retinopathy grade R3) are to be seen within 2 , routinely referred patients within 10 weeks.

in Fig. 1. Patients that meet referral criteria to hospital were invited to a clinical appointment in either a traditional F2F or virtual setting triaged according to hospital guidelines (Fig. 1).

Initial assessments in the F2F or virtual settings were carried out as previously described [14, 15]. Briefly, VA and noncontact intraocular pressure were taken by trained nurses and entered into an electronic health record system, either Medisoft (Medisoft Ltd., Leeds, UK) or OpenEyes (OpenEyes Foundation, London, UK). Patients then received dilated fundus photography. Depending on the availability of retinal imaging at the site, either 2-field $45^{\circ}$ fundus photography or wide-field fundus photography (Optos) was obtained. All patients underwent macula OCT volume scan (Topcon 3D OCT scan). In a virtual consultation, a structured history was taken by a trained nurse. History and investigations were remotely reviewed by an ophthalmologist within 1 week with outcome determined as per hospital guidelines (Supplementary Fig. 1). In F2F clinics, the consultation with the retinal specialist took place on the same day as the investigations. Herein, history taking, dilated slit-lamp
At Moorfields Eye Hospital, patients referred by the DESP can initially be seen in a face-to-face (F2F) or virtual-clinic (VC) appointment. This is determined by ophthalmologist-led eminence-based triage guidance. Patients with proliferative retinopathy grade 3 (R3), visual acuity below 61 Early Treatment Diabetic Retinopathy (ETDRS) letters, ungradable fundus imaging in primary care, vulnerable adult, lens or media opacities, pregnant women, or requirement of interpreter services are ineligible for initial appointments in VC. Patients eligible for both F2F and VC are indiscriminately assigned to either based on booking availability. Outcome of initial assessment can be either follow-up in the hospital or discharge back to the DESP.

examination, imaging review, and a management plan were carried out.

\section{Data extraction}

All clinical data were extracted from the initial MEH appointment. VA is reported in ETDRS letters. For each patient, the eye with the greater VA was considered the better-seeing eye. Where VA for both eyes was identical, this value was used for both better- and worse-seeing eye. Diabetic grading was performed according to the national DESP standards [17]. For each patient, the most severe retinopathy and maculopathy grade was taken forward for analysis. Socio-economic deprivation was extrapolated using postcodes of patient residence to identify corresponding decile index of multiple deprivation based on the English Indices of Deprivation 2019 [18].

\section{Outcomes}

The primary study outcome was the proportion of patients who meet hospital guidelines for initial assessment in VC; 
Fig. 2 Flow diagram of patients referred from the diabetic eye screening program (DESP) to Moorfields Eye Hospital (MEH). Patients referred from DESP to MEH were triaged either to a virtual or a traditional face-to-face appointment. All patients referred between January 2015 and December 2018 were included.

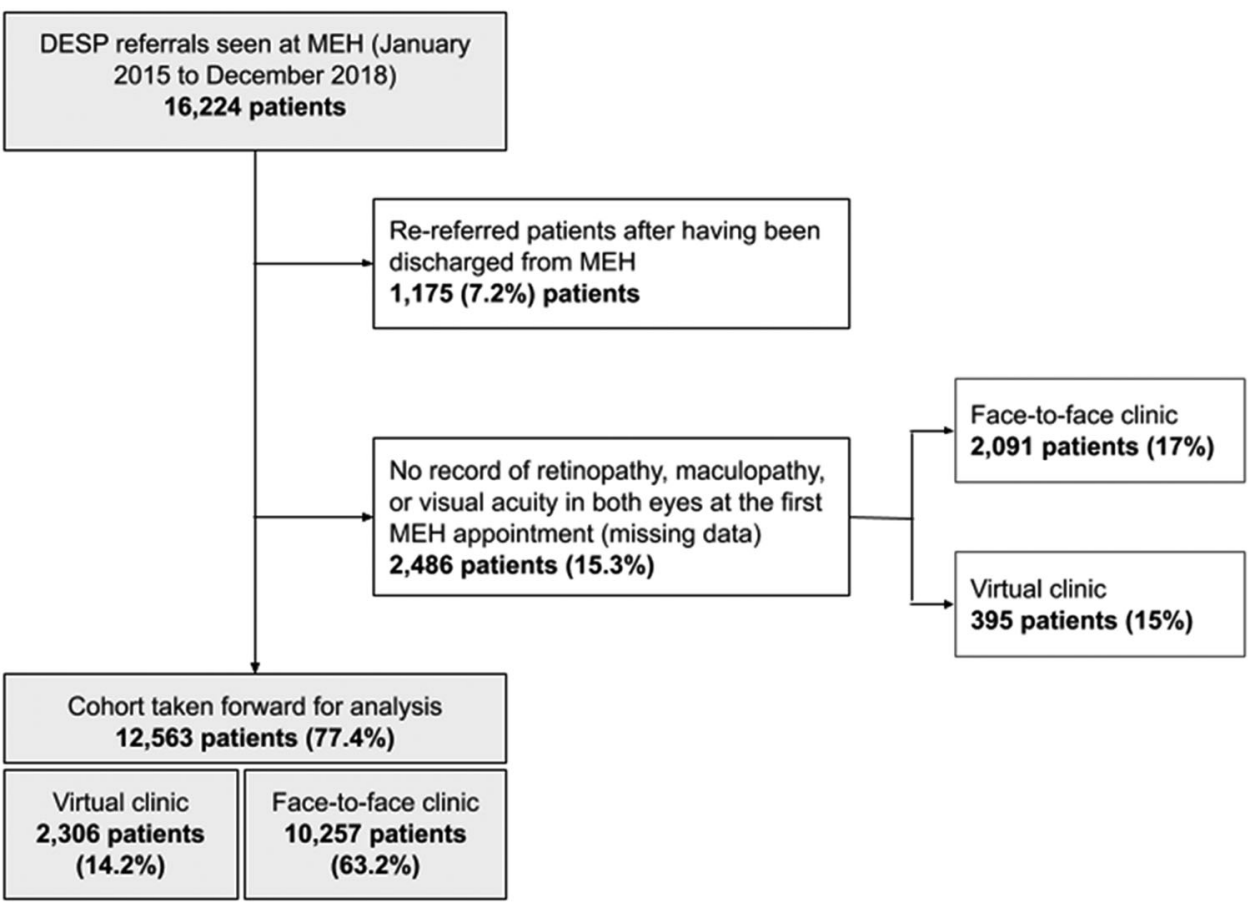

chosen as a surrogate variable for VC adoption and capacity. Secondary outcomes included (i) mean time from the referral of the DESP to the first appointment at the $\mathrm{MEH}$; (ii) mean time from referral of the DESP to discharge; (iii) mean time from referral of the DESP to treatment (either PRP or intravitreal anti-vascular endothelial growth factor)_as surrogate variables for efficiency and safety; (iv) the rate of attendance at the first and if the first has not been attended, second scheduled appointment in F2F compared to virtual clinic (as a surrogate variables for appointment adherence and acceptance); and (v) the distribution of social and economic deprivation indices (as a surrogate variable for accessibility).

\section{Statistical analysis}

Statistical analyses have been carried out in R: a language and environment for statistical computing, R Foundation for Statistical Computing, Vienna, Austria (https://www.Rproject.org/) [19]. Distribution of data was tested by the Shapiro-Wilk normality test. Means of nonparametric groups were compared using Wilcoxon Signed-rank, Wilcoxon Rank-sum, or Kruskal-Wallis tests as appropriate. For more than two groups, multiple pairwise analyses have been carried out using the Wilcoxon Rank-sum test. Calculated means in text and figures are expressed with SD error margin corresponding to the standard deviation, unless otherwise specified. A $p$ value $<0.05$ was considered statistically significant.

\section{Results}

\section{Patient flow, demographics, and clinical features}

From January 2015 to December 2018, there were 16,224 DESP referrals that attended an appointment at $\mathrm{MEH}$. 12,563 patients met the study's selection criteria and were therefore taken forward for analysis (Fig. 2).

We compared demographics between patients that were initially seen in a F2F clinic versus a virtual clinic. A statistically significant difference was not detected in the distribution of gender or mean decile of social and economic deprivation indices. However, statistically significant differences were noted in mean age-those attending a virtualclinic appointment being younger (61.5 years SD 15.0 versus 59.4 years SD 14.4, $p$ value $6.02 \mathrm{e}-10$ ) - and in the distribution of ethnicity. Differences in ethnicity are potentially accounted for by the large proportion of missing data (50.2 and $57.8 \%$ ) seen in both F2F and virtual clinics (Table 1).

Routinely referred patients initially seen in a virtual clinic exhibited a greater mean VA in both the better-seeing (78.7 letters SD 12.2 versus 82.3 letters SD 9.57; $p$ value $<0.001$ ), and the worse-seeing eye (69.4 letters SD 21.8 versus 75.8 letters SD 16.2; $p$ value $<0.001)$ than those seen in a F2F consultation (Table 2). This was expected as current guidance direct patients with VA of $<6 / 18$ Snellen or 61 ETDRS letters to F2F clinics. Similarly, the retinopathy grade R3 is excluded from virtual clinic and therefore features a higher proportion of R0, R1, and R2 (2163 patients, 95\%) than the F2F clinic (8032 patients, 78\%, $p$ value $<0.001$ ). 
Table 1 Demographic information of patients referred by the diabetic eye screening program.
Table 2 Time to the initial appointment and discharge at Moorfields Eye Hospital.
Overall $(n=12563) \quad$ Face-to-face $(n=$ 10257)

Virtual clinic $(n=$

$p$ value

\begin{tabular}{|c|c|c|c|c|}
\hline \multicolumn{5}{|l|}{ Age (years) } \\
\hline Mean (SD) & $61.1(14.9)$ & $61.5(15.0)$ & $59.4(14.4)$ & \multirow[t]{2}{*}{$<0.001$} \\
\hline Median $[\min , \max ]$ & $61.0[15.0,100]$ & $61.0[15.0,100]$ & $59.0[15.0,96.0]$ & \\
\hline \multicolumn{5}{|l|}{ Gender } \\
\hline Female & $5304(42.2 \%)$ & $4302(41.9 \%)$ & $1002(43.5 \%)$ & \multirow[t]{3}{*}{0.258} \\
\hline Male & $7253(57.7 \%)$ & $5951(58.0 \%)$ & $1302(56.5 \%)$ & \\
\hline Unknown & $6(0.0 \%)$ & $4(0.0 \%)$ & $2(0.1 \%)$ & \\
\hline \multicolumn{5}{|l|}{ Ethnicity } \\
\hline South-East Asian & $3130(24.9 \%)$ & $2682(26.1 \%)$ & $448(19.4 \%)$ & \multirow[t]{6}{*}{$<0.001$} \\
\hline Afro Caribbean & $1160(9.2 \%)$ & $907(8.8 \%)$ & $253(11.0 \%)$ & \\
\hline Caucasian & $1641(13.1 \%)$ & $1389(13.5 \%)$ & $252(10.9 \%)$ & \\
\hline Chinese & $43(0.3 \%)$ & $41(0.4 \%)$ & $2(0.1 \%)$ & \\
\hline Mixed & $111(0.9 \%)$ & $94(0.9 \%)$ & $17(0.7 \%)$ & \\
\hline Unknown & $6478(51.6 \%)$ & $5144(50.2 \%)$ & $1334(57.8 \%)$ & \\
\hline \multicolumn{5}{|l|}{ IMD (decile) } \\
\hline Mean (SD) & $4.66(2.25)$ & $4.67(2.22)$ & $4.61(2.37)$ & \multirow[t]{2}{*}{0.26} \\
\hline Median [min, max] & $4.00[1.00,10.0]$ & $4.00[1.00,10.0]$ & $4.00[1.00,10.0]$ & \\
\hline
\end{tabular}

Data from the total cohort presented collectively and sub-stratified by first clinic type: face-to-face or virtual. The mean and standard deviation (SD) of patient age at the initial appointment (baseline), index of multiple deprivation (IMD) decile, and index of deprivation affecting older people (IDAOP) decile were compared. Wilcoxon signed-rank test was used for comparison of face-to-face and virtual-clinics sub-cohorts. The Chisquare test was used for categorical comparison i.e., gender and ethnicity.

\begin{tabular}{|c|c|c|c|c|}
\hline & \multicolumn{2}{|l|}{ Face-to-face } & \multicolumn{2}{|l|}{ Virtual clinic } \\
\hline & Routine $(n=8756)$ & Urgent $(n=1436)$ & Routine $(n=2201)$ & Urgent $(n=16)$ \\
\hline \multicolumn{5}{|c|}{ Time from referral to initial appointment (days) } \\
\hline Mean (SD) & $80.9(44.4)$ & $27.9(30.1)$ & $66.9(35.9)$ & $30.9(19.3)$ \\
\hline Median [min, max] & $76.0[0.00,1840]$ & $18.0[0.00,303]$ & $63.0[2.00,406]$ & $21.0[12.0,78.0]$ \\
\hline \multicolumn{5}{|c|}{ First appointment outcome } \\
\hline Follow-up & $7677(87.7 \%)$ & $1308(91.1 \%)$ & $1987(90.3 \%)$ & $13(81.2 \%)$ \\
\hline Discharge & $1079(12.3 \%)$ & $128(8.9 \%)$ & $214(9.7 \%)$ & $3(18.8 \%)$ \\
\hline \multicolumn{5}{|c|}{ Time from referral to discharge (days) } \\
\hline Mean (SD) & $86.3(37.0)$ & $33.8(34.2)$ & $71.7(30.8)$ & $66.0(8.19)$ \\
\hline Median [min, max] & $81.0[0.00,344]$ & $24.5[4.00,248]$ & $70.0[17.0,244]$ & $68.0[57.0,73.0]$ \\
\hline Missing & $7677(87.7 \%)$ & $1308(91.1 \%)$ & $1987(90.3 \%)$ & $13(81.2 \%)$ \\
\hline
\end{tabular}

Mean duration between receiving a patient referral and first attended appointment was calculated and substratified by clinic type (face-to-face and virtual clinic) as well as referral urgency (routine and urgent). For patients whose initial appointment resulted in the clinician's decision to discharge, duration between initial referral and date of discharge decision were similarly extrapolated. SD signifies standard deviation.
In terms of maculopathy grade, we found a statistically significant difference in the distribution of maculopathy grades between $\mathrm{F} 2 \mathrm{~F}$ and virtual clinics (Table $2 ; p$ value $<$ $0.001)$. Indeed, a greater proportion of $\mathrm{M} 0$-graded patients (5463 patients, $53.3 \%$ ) was observed in $\mathrm{F} 2 \mathrm{~F}$ versus 461 patients $(19.9 \%)$ in virtual clinic $(p$ value $<0.001)$. In general, there was a higher proportion of M1 gradings in virtual clinic (413 patients, 17.9\%) than in F2F
(909 patients, 9\%; $p$ value $<0.001$ ). Likewise, there was a higher proportion of M1S-graded patients in virtual (1290 patients, $58.1 \%$ ) versus F2F clinics (2586 patients, $25.3 \% ; p$ value $<0.001)$. Complying with local triage guidance, we found higher proportions of patients with a maculopathy grade of M1A in F2F (654 patients, 7.8\%) than in virtual clinic (57 patients, $2.5 \%, p$ value $<0.001$ ) (Tables 1, 2, and Fig. 3). 

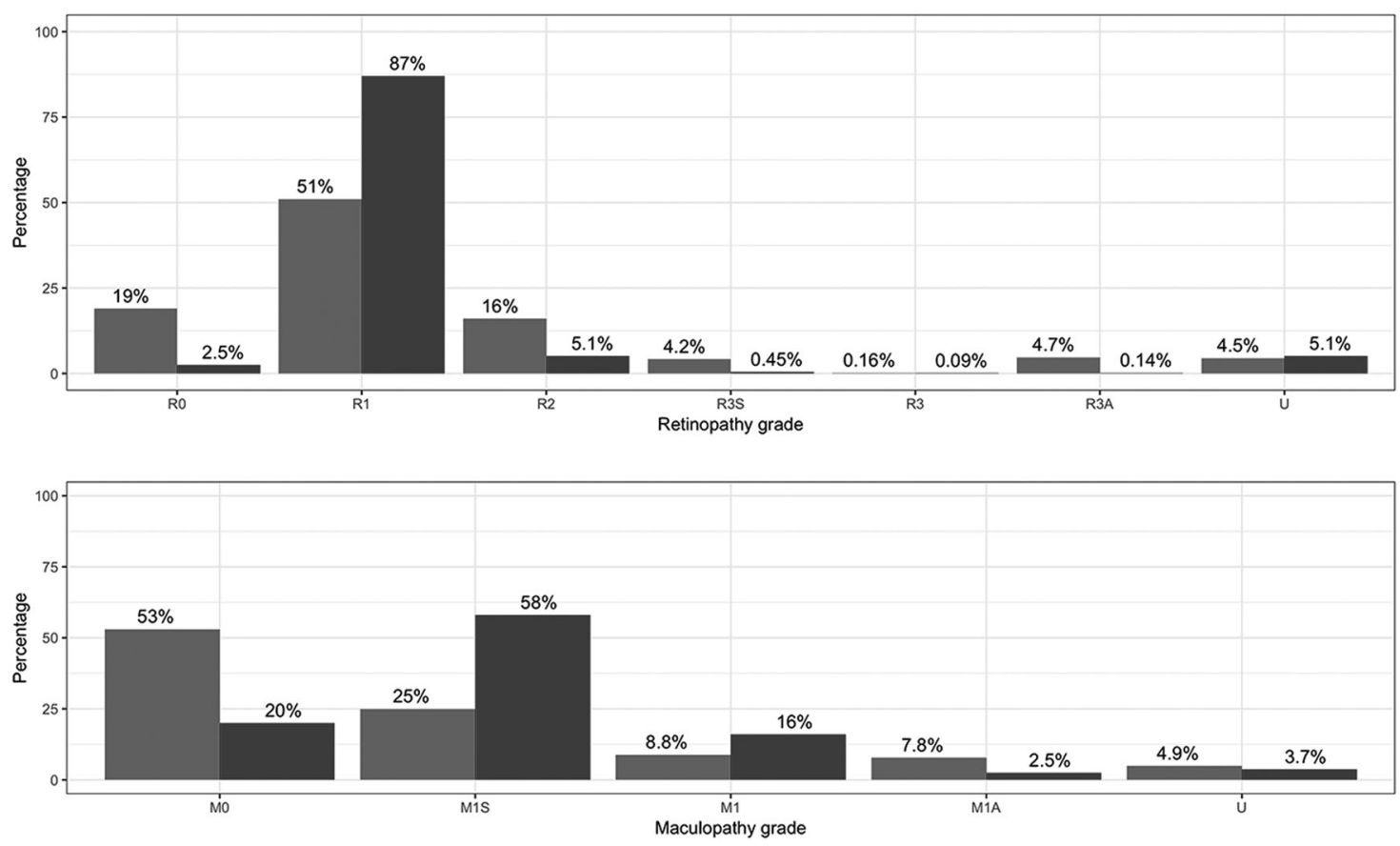

Fig. 3 Clinical features of patients sub-stratified by clinic type of initial assessment. Distribution of retinopathy (upper panel), maculopathy (lower panel) grades, and ungradeable (U) expressed as a proportion of total referrals initially seen in the face-to-face (blue).

\section{Virtual-clinic adoption and capacity}

Current MEH guidance directs a subset of DESP referrals (retinopathy grade R3 or VA below or equal 6/18 Snellen or 61 Letters) for initial assessment in F2F clinics; patients can otherwise indiscriminately be seen in either a F2F or a virtual clinic. Of all patients included in this study, 8833 (70.7\%) met the criteria for a virtual consultation. Similarly, $6253(71.4 \%)$ of routine referrals initially seen in the F2F setting met the criteria for virtual consultation (Fig. 4a).

A considerable proportion of routine referrals that were discharged on the initial F2F appointment also met triage criteria for virtual clinics (815 patients, $75.6 \%$ ). Notably, the proportion of initial appointments taking place in a virtual setting has progressively increased over the past years; from $4 \%$ in 2015 to $24 \%$ in 2018. Still, the majority of patients in 2018 are initially assessed F2F (Fig. 5).

\section{Attendance rate of virtual versus F2F consultations}

We queried whether the smaller proportion of referred patients initially assessed in virtual clinic reflected a greater reluctance from patients to attend a virtual than a F2F consultation. Of all initial invitations to a MEH appointment, $32 \%$ (5076 of 15,944) and 28\% (994 of 3550) did not attend their booked F2F or virtual consultation, respectively. For the sub-cohort that did not attend their initial appointment, a similar trend was apparent for the second appointment as 55\% (2282 of 4177) and 52\% (446 of 853) did not attend following an invitation to a F2F or a virtual consultation, respectively.

\section{Clinical decisions at the first appointment}

Patients referred by the DESP seen in HES can be substratified into those: (i) where management by secondary services is not indicated and therefore they are immediately discharged for DESP surveillance; (ii) that require treatment or active monitoring by secondary care. Of all routine DESP referrals, a greater proportion was discharged following initial assessment in a F2F (1085 patients, 12.3\%) than in a virtual consultation (224 patients, 9.9\%).

Triage guidelines divert patients with proliferative retinopathy and active maculopathy to F2F clinics. As such, a greater proportion of patients initially seen in F2F clinics was indicated for PRP (routinely referred: 278 patients, 3\%; urgently referred: 342 patients, $4 \%$; Supplementary Table 1) or intravitreal injection of anti-VEGF (routinely referred: 135 patients, $2 \%$; urgently referred: 54 patients, $1 \%$; Supplementary Table 2$)$ than in virtual clinics $(0.1 \%$ laser; $1 \%$ injection; Supplementary Table 3).

\section{Time from referral acceptance to appointment, discharge, and treatment}

When compared with F2F, we observed a mean time from routine referral to initial assessment to be shorter for virtual clinics (68.0 days SD 37.3 versus 80.9 days SD 44.5, 

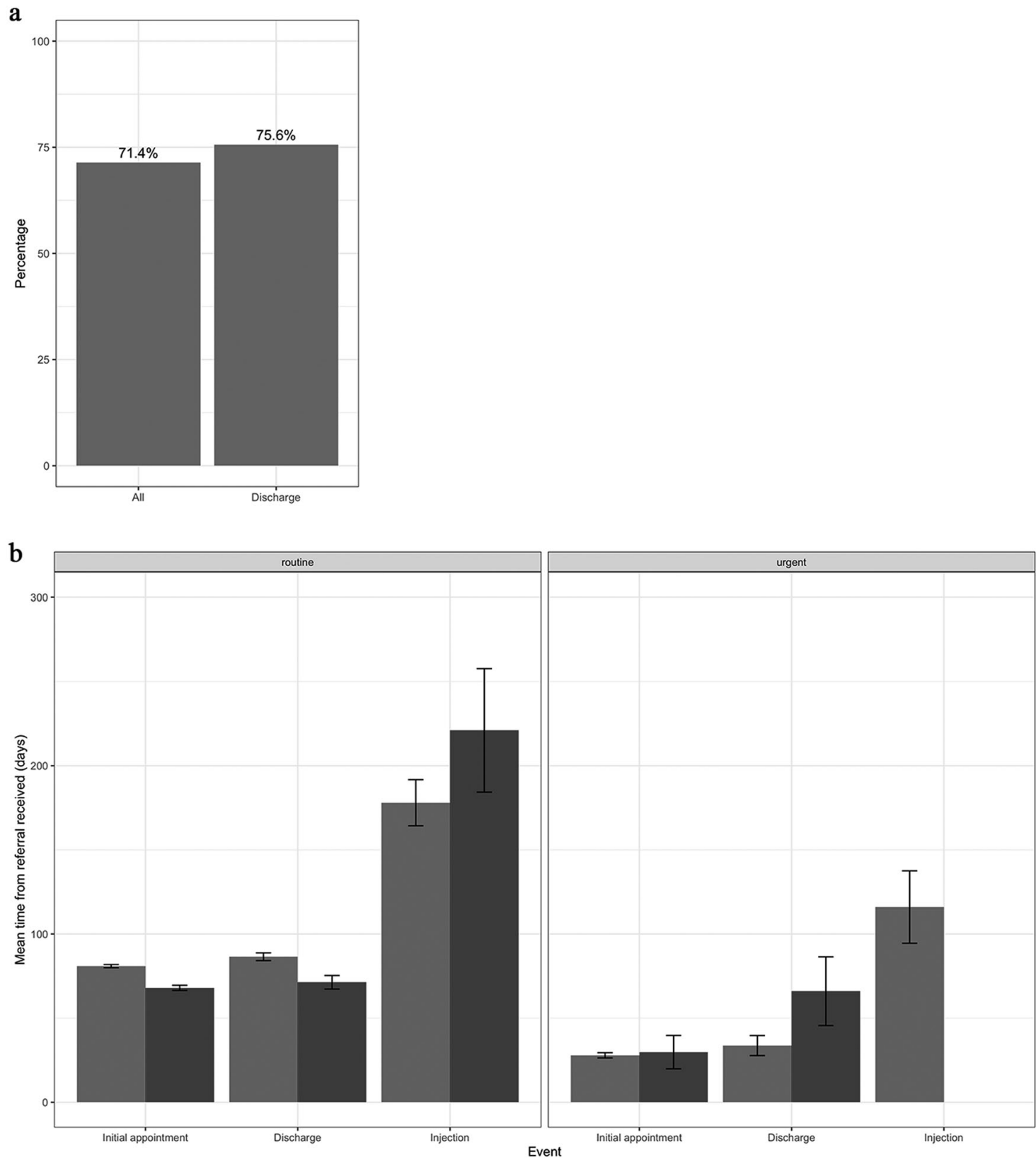

Fig. 4 Current face-to-face first appointments that meet the virtual clinic criteria anad a comparison of outcomes from both clinics. a Proportion of routine face-to-face appointments that meet current triage criteria for assessment in virtual clinic. b A comparison of initial clinic type (face-to-face [blue] and virtual retina clinic [red]) in terms

of mean duration from receiving patient referral to: initial appointment, discharge decision, receiving initial intravitreal injection. This was further stratified by urgency of referral, routine (left panel), and urgent (right panel). Error bars signify standard deviation.

$p$ value $<0.001)$. Consequently, the decision to discharge a patient back to the primary care at the first appointment (when indicated) was also made sooner in virtual clinics (71.3 days SD 30.5 versus 86.5 days SD 38.6; $p$ value $<$ $0.001)$.

Consistent with MEH guidelines, urgent DESP referrals (11.6\% of all referrals) were largely seen in a F2F clinic $(1454 / 1461 ; 98.9 \%)$. All 17 urgent referrals to a virtual clinic were due to clerical error i.e., patients were clinically appropriate for routine referral, but accidently marked as urgent. Nevertheless, there was no statistically significant difference in the mean time from referral to appointment attendance between F2F (27.9 days SD 30.2) and virtual clinic (29.8 days SD 19.3; $p$ value 0.95 ).

Of routine referrals that underwent intravitreal injections, the clinic type of the first appointment did not affect the mean time from referral to receiving treatment (VC 220.7 days SD 84.8 versus F2F clinics 178.0 days SD 80.7, 
Fig. 5 Overview of patients referred by the diabetic eye screening program to Moorfields Eye Hospital. Total number of patients from the diabetic eye screening program (DESP) that are referred to and seen at Moorfields Eye Hospital per annum (blue; left axis). Patients are initially assessed in either a face-to-face or virtual consultation. Proportion of initial assessments that are virtual versus face-to-face is indicated in red (right axis).

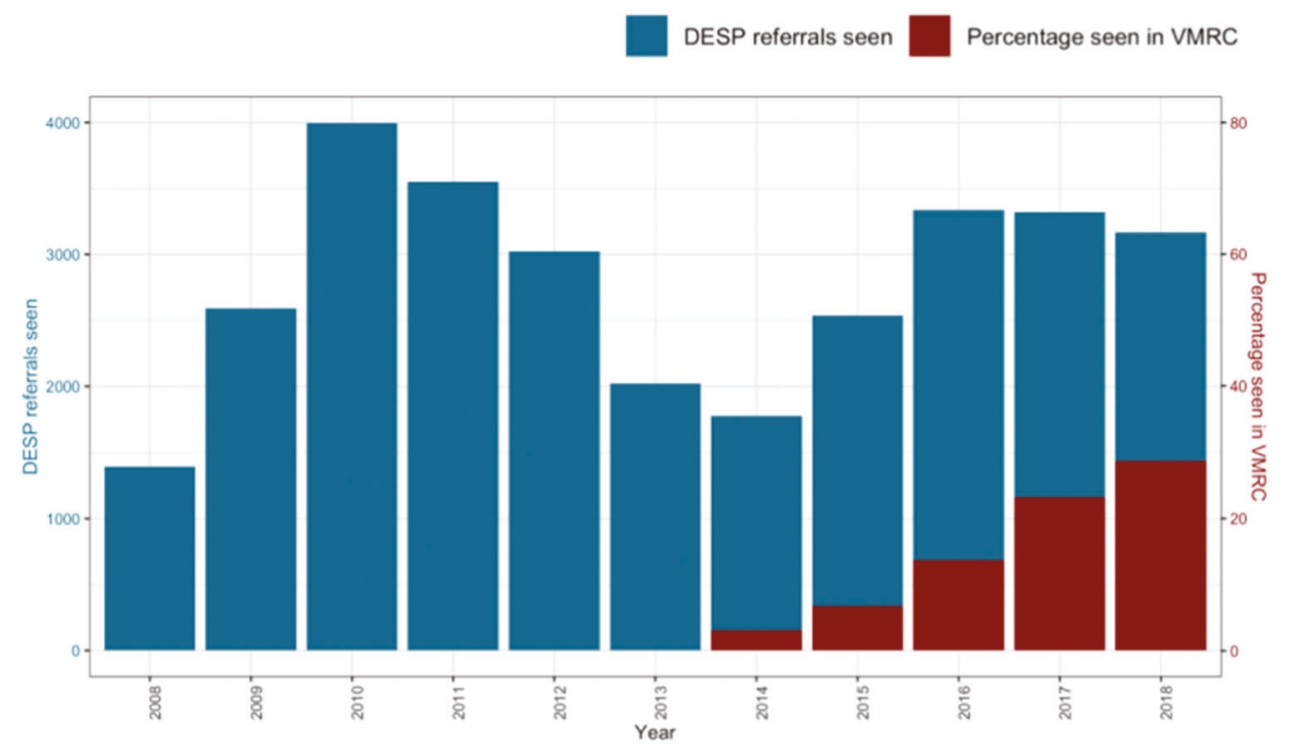

$p$ value 0.056) (Fig. 4b and Supplementary Table 4). Of all DESP referrals initially seen F2F, 619 patients (6\%) underwent PRP (Fig. 4b and Supplementary Table 4). Here, the mean duration from referral to treatment was 94.3 days (SD 50.1) for routine and 35.3 days (SD 28.2) for urgent referrals. Of patients referred to virtual clinics, only five presented with a retinopathy grade that potentially warranted PRP (R3 or R3A). Two received PRP within 145 days of the referral acceptance as the others had previous treatment.

\section{Discussion}

\section{Main findings}

This study sought to evaluate the potential of a combined F2F- and virtual-clinic system at MEH over a 4-year observation period. Notably, only a fifth of all referred patients was initially assessed in a virtual consultation. However, nearly three quarters of all patients initially seen in a F2F clinic were suitable for a virtual consultation in terms of retinopathy grade and visual function.

In terms of efficiency, DESP referrals triaged to virtual clinics were able to attend their first HES appointment 2 weeks sooner (on average) than if triaged to a F2F clinic. Consequently, the time to clinical decision was also faster for patients discharged back to the DESP at the first appointment. Hence, patients that did not require secondary care spent less time in the HES when managed in a virtual clinic. This gain in efficiency did not adversely affect patient safety. We did not observe a statistically significant difference in the mean time from referral acceptance to urgent appointments or intravitreal injections between the two clinic types. In addition, our findings suggest that patient acceptability and accessibility of virtual clinics were uncompromised. That is, a statistically significant difference in attendance rates or indices of socio-economic deprivation between patients assessed in virtual versus F2F clinics was not detected.

\section{Contextualising findings and their implications}

Eminence-based hospital guidance directs patients referred by the DESP with suspicion of severe retinal disease to a F2F clinic. The intention here is to minimise and prevent unnecessary delays between referral and potential visionpreserving treatment $[20,21]$. Yet, we did not observe a statistically significant difference in the mean duration from urgent referral to appointment in F2F and virtual clinics; despite all urgent referrals to virtual clinic being clerical errors. A statistically significant difference was also not detected in the mean duration between routine referrals and intravitreal injection when comparing $\mathrm{F} 2 \mathrm{~F}$ and virtual consultation. This is likely because patients requiring treatment are booked into a specialised interventional clinic (PRP for proliferative retinopathy and intravitreal antiVEGF injections for maculopathy) regardless of whether they are assessed virtually or F2F. However, we were unable to compare the mean time to PRP as this was only indicated in two referrals assessed in the virtual setting.

Larger numbers will be required for adequate statistical comparison. If these preliminary analyses are confirmed by adequately powered prospective studies, a revision of current triage guidelines toward inclusion of patients with a higher risk profile for sight-threatening disease into virtual clinics may be warranted. Our preliminary results suggest that the modest adoption of virtual clinics reflect a capacity 
constraint of the current infrastructure in the hospital service, rather than patients' reluctance in regards to virtual consultations.

At present, the medical retina virtual-clinic capacity (1000 per annum) is unable to match the referral rate from DESP to MEH (circa 4300 per annum). However, upscaling of virtual-clinic capacity has been accomplished for other ophthalmic services, including the MEH glaucoma service wherein all new patients referred by primary care (circa 5000 per annum) are first assessed in an analogous virtual consultation [13, 22, 23]. Certainly, the demand for telemedicine solutions has taken centre stage with pressures from the current COVID-19 climate to deliver clinical care with minimal time spent in close proximity. Key factors required for the expansion of the service capacity include training of qualified graders, automating administrative and failsafe processes, and utilising community imaging devices, such as OCT, where available. Moreover, cloud-based platforms that are robust, configurable, and interoperable will be imperative to the success of scaling these teleophthalmology solutions. In light of current national efforts to rapidly scale up telemedicine services (e.g., NHS England extending Attend Anywhere and Heath at Home to over 200 providers), the stage has been set for post hoc analyses that will further our understanding of efficacy, safety, upscaling, and maintenance of these innovations.

\section{Strengths and limitations}

To the best of our knowledge, this is the first retrospective cohort study to report on the efficiency, safety, and acceptability of a combined F2F- and virtual-clinic system for patients referred from a national diabetic eye screening service.

Our study has several limitations. While we found that about three-fourths of patients seen in F2F clinic would have been suitable for a virtual consultation in terms of severity grading and visual function, we could not exclude that they were triaged to a F2F clinic because of the need for interpreter service, vulnerability, or pregnancy. Moreover, we performed our analyses by taking the most severe retinopathy and maculopathy grade for each patient, which means that the combinations of retinopathy and maculopathy gradings does not reflect true constellations that occurred in a study eye.

\section{Conclusion}

This study presents preliminary evidence that suggests that virtual consultations are a safe and efficacious alternative to traditional appointments for patients referred by the national diabetic eye screening service. Particularly for those carrying low risk for sight-threatening disease and good visual prognosis. However, its potential is limited by the current lack of resources required for expansion. We envisage further research in this arena to focus on automation of image analysis and clinical decision making, as well as, the crucial administrative processes that enable the delivery of a robust teleophthalmology service.

\section{Summary}

\section{What was known before}

- The UK diabetic eye screening service has strained capacity of HES.

\section{What this study adds}

- Virtual consultation is an appropriate alternative to traditional F2F consultations for a substantial proportion of referrals from the UK diabetic eye screening service.

- Its implementation can alleviate service demands without diminishing quality of clinical care.

\section{Compliance with ethical standards}

Conflict of interest The authors declare that they have no conflict of interest.

Publisher's note Springer Nature remains neutral with regard to jurisdictional claims in published maps and institutional affiliations.

\section{References}

1. Cho NH, Shaw JE, Karuranga S, Huang Y, da Rocha Fernandes JD, Ohlrogge AW, et al. IDF diabetes Atlas: global estimates of diabetes prevalence for 2017 and projections for 2045. Diabetes Res Clin Pr. 2018;138:271-81.

2. Leese GP, Stratton IM, Land M, Bachmann MO, Jones C, Scanlon $\mathrm{P}$, et al. Progression of diabetes retinal status within community screening programs and potential implications for screening intervals. Diabetes Care. 2015;38:488-94. https://doi. org/10.2337/dc14-1778.

3. Liew G, Michaelides M, Bunce C. A comparison of the causes of blindness certifications in England and Wales in working age adults (16-64 years), 1999-2000 with 2009-2010. BMJ Open. 2014:4:e004015.

4. Scanlon PH. The English National Screening Programme for diabetic retinopathy 2003-2016. Acta Diabetol. 2017;54:515-25.

5. Anon. NHS Screening Programmes-diabetic eye screening. https://assets.publishing.service.gov.uk/government/uploads/ system/uploads/attachment_data/file/493991/DES_-_revision_ 2.pdf. Accessed 2 Nov 2019.

6. Looker HC, Nyangoma SO, Cromie DT, Olson JA, Leese GP, Black MW, et al. Rates of referable eye disease in the Scottish National Diabetic Retinopathy Screening Programme. Br J 
Ophthalmol. 2014;98:790-5. https://doi.org/10.1136/bjophtha lmol-2013-303948.

7. The Royal College of Ophthalmologists. Increasing demand on hospital eye services risks patients losing vision. The Royal College of Ophthalmologists; 2016. https://www.rcophth.ac.uk/ 2016/03/increasing-demand-on-hospital-eye-services-risks-pa tients-losing-vision/. Accessed 2 Nov 2019.

8. Silva PS, Cavallerano JD, Aiello LM, Aiello LP. Telemedicine and diabetic retinopathy: moving beyond retinal screening. Arch Ophthalmol. 2011;129:236-42.

9. D'souza Y, Tufail A. The role of telemedicine in improving the referral service for consideration of treatment for age-related macular degeneration in a tertiary referral centre. Acta Ophthalmologica Scand. 2007;85. 10.1111/j.1600-0420.2007.01062_3313.x.

10. Scanlon PH, Aldington SJ, Stratton IM. Delay in diabetic retinopathy screening increases the rate of detection of referable diabetic retinopathy. Diabet Med. 2014;31:439-42.

11. Kiernan DF, Mieler WF, Hariprasad SM. Spectral-domain optical coherence tomography: a comparison of modern high-resolution retinal imaging systems. Am J Ophthalmol. 2010;149:18-31.

12. Silva PS, Cavallerano JD, Sun JK, Noble J, Aiello LM, Aiello LP. Nonmydriatic ultrawide field retinal imaging compared with dilated standard 7-field 35-mm photography and retinal specialist examination for evaluation of diabetic retinopathy. Am J Ophthalmol. 2012;154:549-59.e2. https://doi.org/10.1016/j.ajo.2012.03.019.

13. Kotecha A, Brookes J, Foster P, Baldwin A. Experiences with developing and implementing a virtual clinic for glaucoma care in an NHS setting. Clin Ophthalmol. 2015:1915. https://doi.org/10. 2147/opth.s92409.

14. Kortuem K, Fasler K, Charnley A, Khambati H, Fasolo S, Katz M, et al. Implementation of medical retina virtual clinics in a tertiary eye care referral centre. Br J Ophthalmol. 2018;102:1391-5.
15. Kern C, Kortuem K, Hamilton R, Fasolo S, Cai Y, Balaskas K, et al. Clinical outcomes of a hospital-based teleophthalmology service: what happens to patients in a virtual clinic? Ophthalmol Retin. 2019;3:422-8.

16. Vandenbroucke JP, von Elm E, Altman DG, Gøtzsche PC, Mulrow $\mathrm{CD}$, Pocock SJ, et al. Strengthening the reporting of observational studies in epidemiology (STROBE): explanation and elaboration. Int J Surg. 2014;12:1500-24.

17. Harding S, Greenwood R, Aldington S, Gibson J, Owens D, Taylor R, et al. Grading and disease management in national screening for diabetic retinopathy in England and Wales. Diabet Med. 2003;20:965-71.

18. Anon. English indices of deprivation 2019. https://www.gov.uk/ government/statistics/english-indices-of-deprivation-2019. Accessed 31 Jan 2020

19. R Core Team. R: a language and environment for statistical computing. R Foundation for Statistical Computing. 2018. http://www.R-project.org/.

20. Anon. Indications for photocoagulation treatment of diabetic retinopathy: Diabetic Retinopathy Study Report no. 14. The Diabetic Retinopathy Study Research Group. Int Ophthalmol Clin. 1987;27:239-53.

21. Flaxel CJ, Adelman RA, Bailey ST, Fawzi A, Lim JI, Vemulakonda $\mathrm{GA}$, et al. Diabetic retinopathy preferred practice pattern ${ }^{\circledR}$ Ophthalmology. 2019. https://doi.org/10.1016/j.ophtha.2019.09.025.

22. Vardy J, Jenkins PJ, Clark K, Chekroud M, Begbie K, Anthony I, et al. Effect of a redesigned fracture management pathway and 'virtual' fracture clinic on ED performance. BMJ Open. 2014;4: e005282. https://doi.org/10.1136/bmjopen-2014-005282.

23. Jayaram PR, Bhattacharyya R, Jenkins PJ, Anthony I, Rymaszewski LA. A new 'virtual' patient pathway for the management of radial head and neck fractures. J Shoulder Elb Surg. 2014;23:297-301. 